\title{
Mapping Strain and Relaxation in 2D Heterojunctions with Sub-picometer Precision
}

Yimo Han ${ }^{1}$, Kayla Nguyen ${ }^{1}$, Michael Cao ${ }^{1}$, Paul Cueva ${ }^{1}$, Mark W. Tate ${ }^{2}$, Prafull Purohit ${ }^{2}$, Saien Xie ${ }^{3}$, Ming-Yang $\mathrm{Li}^{4}$, Lain-Jong $\mathrm{Li}^{4}$, Jiwoong Park ${ }^{3}$, Sol M. Gruner ${ }^{2,5,6,7}$ and David A. Muller ${ }^{1,6}$

1. School of Applied and Engineering Physics, Cornell University, Ithaca, NY.

2. Laboratory of Atomic and Solid State Physics, Cornell University, Ithaca, NY.

3. Department of Chemistry, Institute for Molecular Engineering, and James Franck Institute, University of Chicago, Chicago, IL.

4. Physical Science and Engineering Division, King Abdullah University of Science and Technology, Thuwal, Kingdom of Saudi Arabia.

5. Physics Department, Cornell University, Ithaca, NY.

6. Kavli Institute at Cornell for Nanoscale Science, Ithaca, NY.

7. Cornell High Energy Synchrotron Source, Cornell University, Ithaca, NY.

The electronic, optical, and mechanical properties of two-dimensional (2D) materials are sensitive to, and readily tuned by, strain fields that can be made an order of magnitude larger than in their bulk counterparts. This is especially true in epitaxial lateral heterojunctions, where two different 2D materials seamlessly merge despite a finite lattice mismatch. For small fields of view, geometric phase analysis (GPA) based on atomic-resolution imaging, is able to map the strain field around individual features such as dislocation cores or embedded channels. Fig. 1 shows GPA strain and rotation maps of the sub-nanometer $\mathrm{MoS}_{2}$ channels embedded within a $\mathrm{WSe}_{2}$ monolayer that provide 1D wires in a 2D material [1]. The channels grow from misfit dislocations at the original $\mathrm{MoS}_{2} / \mathrm{WSe}_{2}$ interface, and the channel sidewalls are dislocation-free, displaying large uniaxial strain along the channel direction needed for atomic coherence.

However, for lateral heterostructures where the region of interest span more than a few hundred nanometers, GPA becomes impractical. To decouple the spatial resolution from the strain mapping precision, we developed an approach based on scanning nanobeam diffraction (NBD) to map the strain in graded $\mathrm{WS}_{2}-\mathrm{WSe}_{2}$ lateral heterojunctions with sub-picometer precision (Fig. 2a) [2]. At each position, the full diffraction pattern is collected by our fast $(0.86 \mathrm{~ms}$ readout $)$, high dynamic range $\left(10^{6}: 1\right)$, high sensitivity detector - the electron microscope pixel array detector (EMPAD) [3]. Despite the weak scattering from monolayers, full 4D datasets are collected in 1-2 minutes, with an unsaturated primary beam and well-defined diffracted beams (Fig. 2b). The high signal quality enables precise measurement and analysis of the local lattice constant, strain, and rotations (Fig. 2c-f). The histogram from a nominally flat region in Fig. 2c follows a normal distribution with $0.3 \mathrm{pm}$ standard deviation, indicating that this method has a lattice precision better than $0.3 \mathrm{pm}$, with the local sample distortions placing an upper limit on the spread [2].

While we expect narrow junctions to remain coherent and wide junctions to relax, the relaxation mechanism in 2D films is observed to be different to 3D materials. Fig. 2 shows that the strain is released in wide heterojunctions through both misfit dislocations and out-of-plane ripples, with a lower density of misfits than would be expected from the $4.5 \%$ lattice mismatch. In contrast, the narrow junctions exhibit uniaxial strain parallel to the interface with few dislocations and with only minor ripples observed, and accounting for a very small portion of the strain relaxation. The out-of-plane ripples in the $2 \mathrm{D}$ strain superlattice were mapped using the second moments of the diffraction spots (Fig. 2g,h), exploiting their cone-shaped diffracting beams from the microscopic corrugations [2]. 


\section{References:}

[1] Y. Han et al, Nature Materials 17 (2018), p. 129.

[2] Y. Han et al, arXiv:1801.08053 (2018).

[3] M. W. Tate et al, Microscopy and Microanalysis 22 (2016), p. 237.

[4] Funded by the Cornell Center for Materials Research, an NSF MRSEC (DMR-1719875).

a
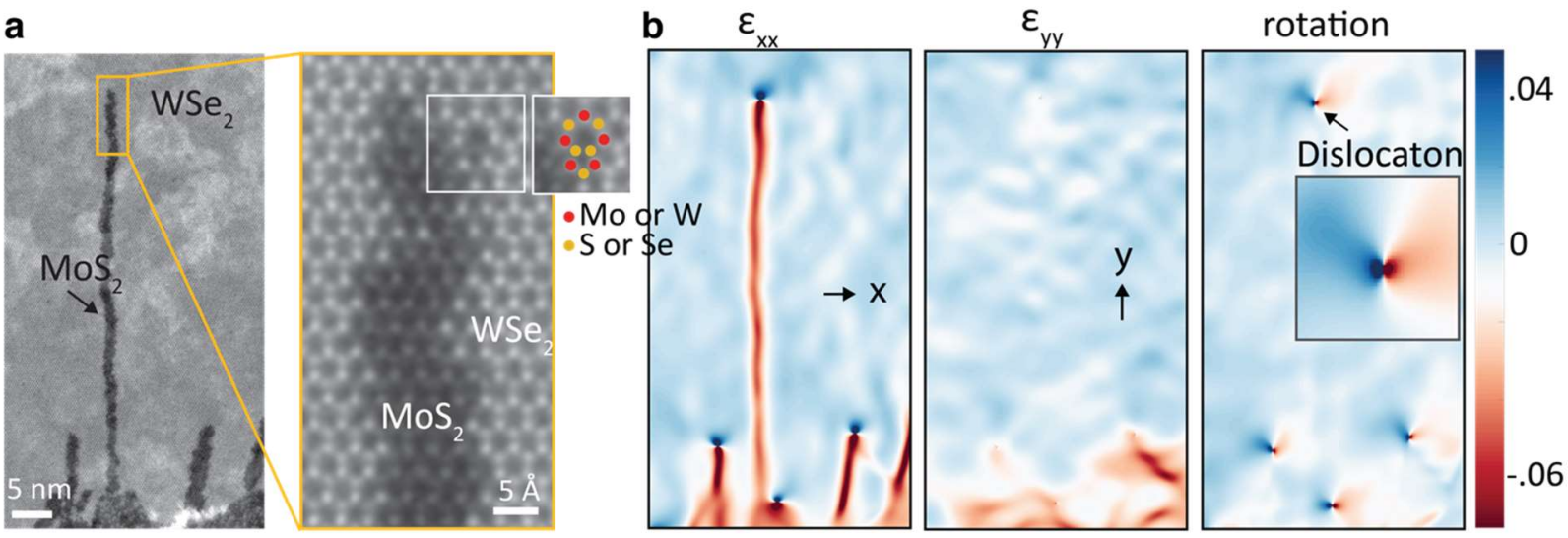

Figure 1. Strain from GPA. (a) Atomic resolution ADF-STEM image of $\mathrm{MoS}_{2} 1 \mathrm{D}$ channels embedded within $\mathrm{WSe}_{2}$ monolayer. The channel ends with the 5|7 membrane dislocation (white box). (b) GPA of the $\mathrm{MoS}_{2}$ channels in (a) with uniaxial strain and rotation components.
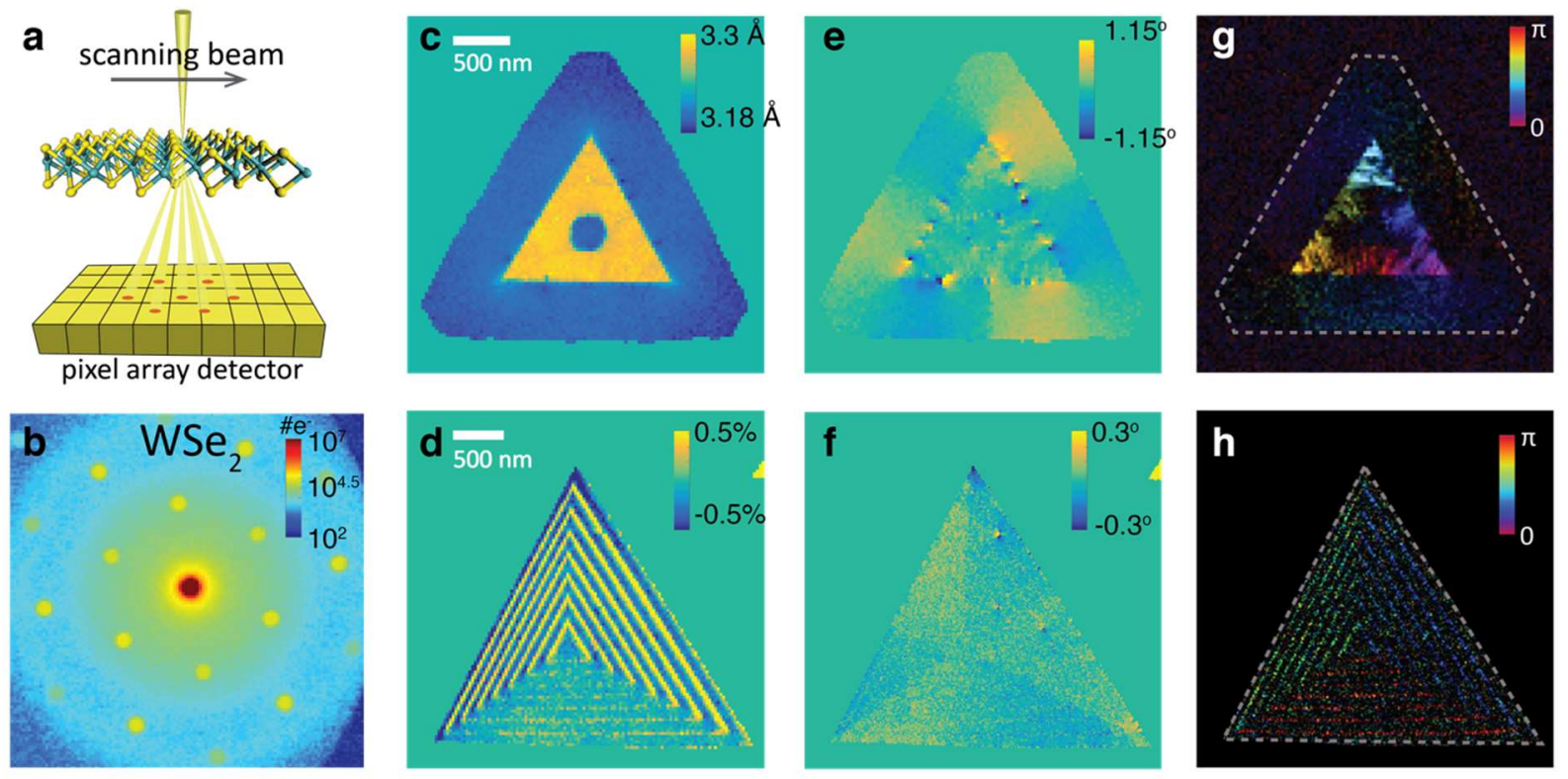

Figure 2. Strain from EMPAD. (a) EMPAD schematic. (b) A full diffraction pattern taken by EMPAD including the unsaturated primary beam and clearly resolved diffraction beams. (c, e, f) Lattice constant, rotation and ripple maps of wide lateral heterojunctions showing misfit dislocations at the interface (e) and ripples relax the lattice mismatch. $(\mathrm{d}, \mathrm{f}, \mathrm{h})$ Uniaxial strain, rotation and ripple maps of narrow lateral heterojunctions, showing coherent strain. 